\title{
Social Distancing as a Population Game in Networked Social Environments
}

\author{
Zhijun $\mathrm{Wu}^{*}$ \\ Department of Mathematics, Iowa State University, Ames, Iowa 50011, USA.
}

Received 4 July 2020; Accepted 10 November 2020

\begin{abstract}
While social living is considered to be an indispensable part of human life in today's ever-connected world, social distancing has recently received much public attention on its importance since the outbreak of the coronavirus pandemic. In fact, social distancing has long been practiced in nature among solitary species, and been taken by human as an effective way of stopping or slowing down the spread of infectious diseases. Here we consider a social distancing problem for how a population, when in a world with a network of social sites, decides to visit or stay at some sites while avoiding or closing down some others so that the social contacts across the network can be minimized. We model this problem as a population game, where every individual tries to find some network sites to visit or stay so that he/she can minimize all his/her social contacts. In the end, an optimal strategy can be found for everyone, when the game reaches an equilibrium. We show that a large class of equilibrium strategies can be obtained by selecting a set of social sites that forms a so-called maximal $r$-regular subnetwork. The latter includes many well studied network types, which are easy to identify or construct, and can be completely disconnected (with $r=0$ ) for the most strict isolation, or allow certain degrees of connectivities (with $r>0$ ) for more flexible distancing. We derive the equilibrium conditions of these strategies, and analyze their rigidity and flexibility on different types of $r$-regular subnetworks. We also extend our model to weighted networks, when different contact values are assigned to different network sites.
\end{abstract}

AMS subject classifications: 92D30, 92D25, 91A22, 91A43, 90C35, 90C27

Key words: Social distancing, epidemic and pandemic prevention, population games, social networks, regular networks, optimal distancing strategies, rigidity, flexibility, and fragility of distancing strategies.

\section{Introduction}

Humans participate in all sorts of social activities, especially in modern societies. We go to school, go to work, attend meetings, go shopping, go to restaurants, watch shows,

${ }^{*}$ Corresponding author. Email address: zhijun@iastate.edu (Z. Wu) 
movies, or sports, etc. The world is formed by a huge number of social sites that host these activities. These sites are also closely connected for people to contact, meet, and socialize across. The world is a well-connected network. People's daily life is simply a busy agenda of events at different event sites in this network, with different visiting frequencies for different sites. As such, the world can be modeled as a social network, with the nodes representing the social sites and the links the connections among the social sites. The social life of an individual can be described by the frequencies of the individual to visit these social sites, and the social behavior of the population can be described by these frequencies averaged over the whole population.

Mathematically, we can represent a social network with a graph $G=(V, E)$, where $V=$ $\{1, \cdots, n\}$ is a set of nodes corresponding to the social sites of the network, and $E=\{(i, j)$ : $i$ and $j$ connected $\}$ a set of links between the nodes representing the social connections among the social sites. Let $x \in R^{n}, x \geq 0, \Sigma_{i} x_{i}=1$, be a vector describing the social behavior of an individual on $G$, with $x_{i}$ being the frequency of the individual to visit or stay at node $i$ of $G$. Then, we can define a vector $y \in R^{n}, y \geq 0, \Sigma_{i} y_{i}=1$, for the social behavior of the whole population, with $y_{i}$ being the average frequency of the population to visit or stay at node $i$ of $G$. Let $A$ be the adjacency matrix of $G, A_{i, j}=1$ if $(i, j) \in E, A_{i, j}=0$ if $(i, j) \notin E$, and $A_{i, i}=\alpha \in[0,1]$ for all $i=1, \cdots, n$. Then, in a $y$-population, the amount of social contacts an $x$-individual can make at node $i$ must be $x_{i} A_{i, i} y_{i}$, the contacts with the population on node $i$, plus $x_{i} A_{i, j} y_{j}$, the contacts with the population on other nodes $j$, all together equal to $\Sigma_{j} x_{i} A_{i, j} y_{j}$. Across the network, the social contacts this individual can make must be $\Sigma_{i} \Sigma_{j} x_{i} A_{i, j} y_{j}=x^{T} A y$.

Consider $x$ as the social strategy of an individual, and $y$ the social strategy of the population. Consider $\pi(x, y)=x^{T} A y$ as a payoff function. We can then define a population game where each individual of the population tries to maximize his/her social payoff. The latter can be achieved when an optimal strategy $x^{*}$ is found for every individual. The strategy for the population then becomes $x^{*}$ as well, and a Nash equilibrium is reached. A Nash equilibrium of this game is thus a strategy $x^{*}$ such that

$$
\pi\left(x^{*}, x^{*}\right) \geq \pi\left(x, x^{*}\right), \quad \forall x \in S,
$$

where $S=\left\{x \in R^{n}: \Sigma_{i} x_{i}=1, x_{i} \geq 0, i=1, \cdots, n\right\}$ is the set of all possible social strategies. This game is called a social networking game on network $G$, and has been discussed recently in Wang et al. in 2019 [1]. For social networking, interactions across different social sites are encouraged and the contacts among individuals in the same sites are discounted, and therefore, $A_{i, i}$ are set to zero for all $i=1, \cdots, n$.

Different from social networking, social distancing is to reduce social contacts instead. Therefore, for a given social network, social distancing can be considered as a problem to find a set of social sites in the network where interactions within and between these sites can be minimized. It can therefore be modeled as a population game where each individual tries to minimize his/her social contacts $\pi(x, y)=x^{T} A y$. A Nash equilibrium of this game is then a strategy $x^{*}$ such that

$$
\pi\left(x^{*}, x^{*}\right) \leq \pi\left(x, x^{*}\right), \quad \forall x \in S .
$$




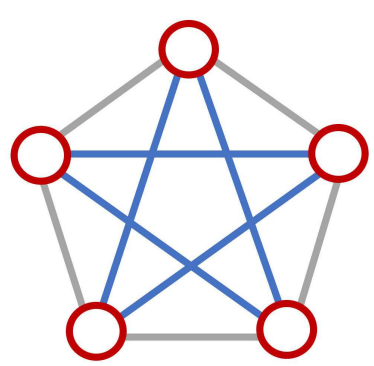

(a)

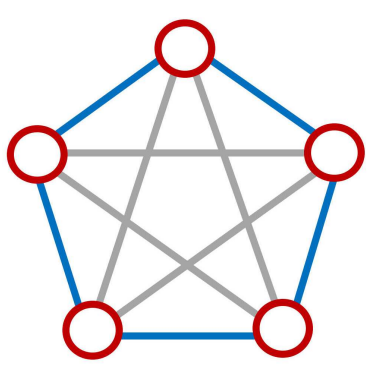

(b)

Figure 1: Social Distancing vs. Social Networking. The complement of network $G=(V, E)$ is a network $\bar{G}=(\bar{V}, \bar{E})$, with $\bar{V}=V$ and $\bar{E}=\{(i, j):(i, j) \notin E\}$. (a) Network $G$ with links colored in blue. (b) Network $\bar{G}$ with links colored in blue. The links in gray are those in the complement of the corresponding network. A social distancing game on $G$ is a social networking game on $\bar{G}$, and vice versa.

We call this game a social distancing game on network $G$. For social distancing, we also count the contacts among individuals in the same sites, and therefore, set $A_{i, i}=1$ for all $i=1, \cdots, n$. Let $\bar{G}=(\bar{V}, \bar{E})$ be the complement of $G$, with $\bar{V}=V$ and $\bar{E}=\{(i, j):(i, j) \notin E\}$. Then, it is easy to see that a social distancing game on network $G$ is equivalent to a social networking game on $\bar{G}$, and vice versa (see Fig. 1).

While social living is considered to be an indispensable part of human life in today's ever-connected world, social distancing has recently received much public attention on its importance since the outbreak of the coronavirus pandemic [2-7]. In fact, social distancing has long been practiced in nature among solitary animals, who always try to distance themselves from others, sometimes for more efficient foraging, sometimes for selfprotection from infightings, and sometimes for avoiding disease infections as well [8-10]. In human societies, social distancing has been recommended by health experts as a special yet effective measure for mitigating the spread of diseases during epidemics or pandemics [11-14]. It can be as simple as just to keep distances from each other, or be more cooperative to follow certain rules such as avoiding large gatherings, canceling events, and closing schools, or to shut down places such as movie theaters, shopping centers, and commercial areas [15,16].

Much work has been done on modeling social distancing in the past [17-27] and especially in this year since the outbreak of the coronavirus pandemic [28-33], most focusing on epidemiological models and predictions, with social distancing as a behavioral factor affecting the spread of the diseases. The impacts of distancing behaviors on contact rates, transmission rates, and hence the infectious rates have been investigated through statistical estimation, dynamic simulation, and model prediction. Our work is focused more on planning of social distancing for a population on how to separate, what to avoid, and where to stay to minimize social contacts and mitigate the spread of the diseases. More specifically, we consider a social distancing problem for how the individuals of a given population, when in a world with a network of social sites, find some sites of the network to visit or stay while avoiding or closing down some others so that the social 
contacts among the individuals across the network can be minimized. This can be critical as a personal decision to make, and perhaps more so as a public health policy to implement. This problem can be modeled as a social distancing game as described above, where every individual tries to find some network sites to visit or stay so that he/she can minimize all his/her social contacts. In the end, an optimal strategy can be found for everyone, when the game reaches an equilibrium. We show that a large class of equilibrium strategies can be obtained by selecting a set of network sites that forms a so-called maximal $r$-regular subnetwork. A regular subnetwork is a subnetwork of all nodes of equal degree. An $r$-regular subnetwork is a subnetwork of all nodes of degree $r$ [34,35] (see Fig. 2). The latter includes many well studied network types, such as the maximal independent set $(r=0)$, the maximal strong matching $(r=1)$, the maximal set of independent cycles $(r=2)$, etc. They are easy to identify or construct, and can be completely disconnected (with $r=0$ ) for the most strict isolation, or allow certain degrees of connectivities (with $r>0$ ) for more flexible distancing. We derive the equilibrium conditions of these strategies, and analyze their rigidity and flexibility on different types of $r$-regular subnetworks. We also extend our model to weighted networks, when different contact values are assigned to different network sites.

Our work do share some important features with other previous studies. For example, Reluga and co-workers $[24,27,36,37]$ have also applied a game dynamic approach to the study of social distancing. Their game involves the investment on social distancing and its effect on the dynamics of SIR populations. Our game is instead for choosing locations for social distancing in a given network of social sites. Eubank et al. [17,38-41] have started investigating network based models in early 2000, and analyzed and simulated large social networks and their impacts on epidemics. Meyers et al. [20,42-45] have led multiple efforts on contact network based epidemic modeling, mostly concerning networks of person-to-person contacts. The networks studied in our work can be considered as a special type of contact networks, where the nodes are locations, and the contacts are made through access to the connected locations.

\section{Optimal distancing strategies}

In the social distancing game on a network, every individual wants to find a network site to distance himself/herself from others. In an attempt, a site with a small number of connections would be a good choice. However, if everyone chooses this site, no one will benefit. Therefore, a strategy is needed to select a set of sites to visit or stay with a certain frequency at each. Let $x^{*} \in S$ be a strategy everyone would like to take, with $x_{j}^{*}$ being the frequency of visiting or staying at site $j$. In effect, the population will distribute on the network also as $x^{*}$, with $x_{j}^{*}$ being the probable fraction on site $j$. With the population distributed as such, the social contacts an individual at site $i$ can make with the population at site $j$ will be $A_{i, j} x_{j}^{*}$. If there is a link between $i$ and $j$, i.e., $A_{i, j}=1, A_{i, j} x_{j}^{*}=x_{j}^{*}$; otherwise, $A_{i, j} x_{j}^{*}=0$. In total, the social contacts this individual can make, if at site $i$, will 


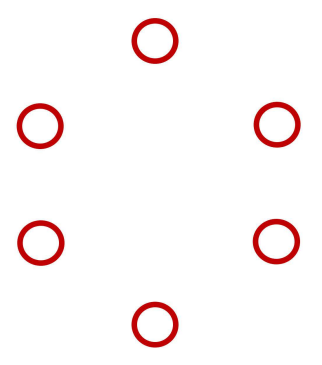

(a)

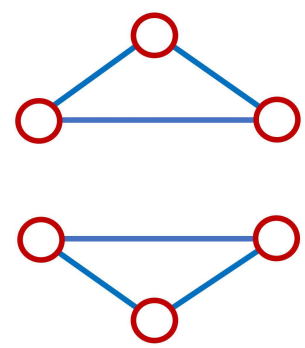

(c)

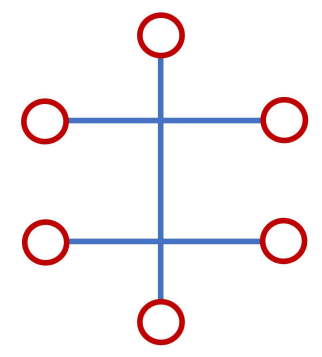

(b)

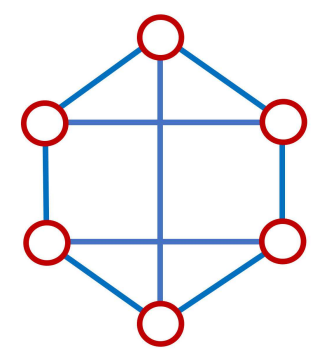

(d)

Figure 2: Regular Networks. A regular network is a network of all nodes of equal degree. An $r$-regular network is a network of all nodes of degree $r$. (a) A 0-regular network. (b) A 1-regular network. (c) A 2-regular network. (d) A 3-regular network.

be $p_{i}\left(x^{*}\right)=\Sigma_{j} A_{i, j} x_{j}^{*}$. The competition among all the sites is then balanced if $p_{i}\left(x^{*}\right)$ are the same, say equal to $\lambda^{*}$, for all $i$ such that $x_{i}^{*}>0$, i.e., for all the sites selected to visit or stay. In addition, $\lambda^{*}$ must be smaller than or equal to $p_{i}\left(x^{*}\right)$ for all $i$ such that $x_{i}^{*}=0$, i.e., for all the sites not selected, for otherwise, by selecting such a site $i$ such that $p_{i}\left(x^{*}\right)<\lambda^{*}$, the individual will be able to reduce the social contacts further. When all these conditions are satisfied, every individual with a strategy $x^{*}$ minimizes all his/her social contacts to $\Sigma_{i} x_{i}^{*} p_{i}\left(x^{*}\right)=\lambda^{*}$, no more, no less, and an equilibrium is reached.

Based on the evolutionary game theory $[46,47]$, we can prove that the above conditions are in fact necessary and sufficient for a strategy $x^{*}$ to be a Nash equilibrium of the social distancing game on a network. We state this property in the following theorem.

Theorem 2.1. Let $A$ be the adjacency matrix of network $G$. Then, a strategy $x^{*} \in S$ is a Nash equilibrium of the social distancing game on $G$ if and only if there is a scalar $\lambda^{*}$ such that $p_{i}\left(x^{*}\right)=$ $\lambda^{*}$ for all $i$ such that $x_{i}^{*}>0$ and $p_{i}\left(x^{*}\right) \geq \lambda^{*}$ for all $i$ such that $x_{i}^{*}=0$, where $p_{i}\left(x^{*}\right)=\Sigma_{j} A_{i, j} x_{j}^{*}$.

Proof. $(\Leftarrow)$ : Assume that there is a scalar $\lambda^{*}$ such that $p_{i}\left(x^{*}\right)=\lambda^{*}$ for all $i$ such that $x_{i}^{*}>0$ and $p_{i}\left(x_{i}^{*}\right) \geq \lambda^{*}$ for all $i$ such that $x_{i}^{*}=0$. Then, $\pi\left(x^{*}, x^{*}\right)=\Sigma_{i} x_{i}^{*} p_{i}\left(x^{*}\right)=\lambda^{*}$. Let $x \in S$ be any strategy. Since $p_{i}\left(x^{*}\right) \geq \lambda^{*}$ for all $i, \pi\left(x, x^{*}\right)=\Sigma_{i} x_{i} p_{i}\left(x^{*}\right) \geq \lambda^{*}$. Therefore, $x^{*}$ is a Nash equilibrium.

$(\Rightarrow)$ : Assume that $x^{*}$ is a Nash equilibrium. Then, $\pi\left(x, x^{*}\right) \geq \pi\left(x^{*}, x^{*}\right)$ for all $x \in S$. Let $\lambda^{*}=\pi\left(x^{*}, x^{*}\right)$. Then, $\pi\left(e_{i}, x^{*}\right) \geq \lambda^{*}$ for all $i$, i.e., $p_{i}\left(x^{*}\right) \geq \lambda^{*}$ for all $i$. It follows that 
$p_{i}\left(x^{*}\right) \geq \lambda^{*}$ for all $i$ such that $x_{i}^{*}=0$. If $p_{i}\left(x^{*}\right)>\lambda^{*}$ for some $i$ such that $x_{i}^{*}>0$, then $\pi\left(x^{*}, x^{*}\right)=\Sigma_{i} x_{i}^{*} p_{i}\left(x^{*}\right)>\lambda^{*}$. Therefore, $p_{i}\left(x^{*}\right)=\lambda^{*}$ for all $i$ such that $x_{i}^{*}>0$.

Let $V^{*}$ be the set of nodes in $G$ selected by a strategy $x^{*}$, i.e., $V^{*}=\left\{i \in V: x_{i}^{*}>0\right\}$. Let $E^{*}$ be the set of links between the nodes in $V^{*}$, i.e., $E^{*}=\left\{(i, j) \in E: i, j \in V^{*}\right\}$. Then, $G^{*}=\left(V^{*}, E^{*}\right)$ is an induced subnetwork of $G$, and is called the supporting subnetwork for $x^{*}$. For an individual, an equilibrium strategy $x^{*}$ on $G^{*}$ then means to visit or stay only at the nodes of $G^{*}$, with a nonzero frequency $x_{i}^{*}$ at node $i$ of $G^{*}$, while for the population, it implies to spread only on the nodes of $G^{*}$, with a nonzero probable fraction $x_{i}^{*}$ on node $i$ of $G^{*}$. In either view, strategy $x^{*}$ with a choice of nodes in $G^{*}$ maximizes the social distances and minimizes the social contacts among all the individuals of the population, if a link between two nodes is counted as a contact while no link as a distance.

The equilibrium strategy for the social distancing game on a network is not necessarily unique. Different equilibrium strategies may have different minimal social contacts. In general, the fewer connections in the supporting subnetwork $G^{*}$, the smaller amount of social contacts at equilibrium. Therefore, an equilibrium strategy without connections in $G^{*}$ can be a first choice for distancing. Such a $G^{*}$ is called an independent set of $G$. It is easy to verify that the larger the independent set, the better the corresponding strategy, when a larger amount of social contacts is minimized. The largest possible independent set that is not contained in another independent set is called a maximal independent set. In fact, a distancing strategy on an independent set of $G$ is not necessarily a Nash equilibrium, but it always is if it is on a maximal independent set of $G$.

Theorem 2.2. Let $x^{*} \in S$ be a strategy for the social distancing game on a network $G=(V, E)$. Let $G^{*}=\left(V^{*}, E^{*}\right)$ be the supporting subnetwork for $x^{*}$. Then, if $G^{*}$ is a maximal independent set of $G, x^{*}$ is a Nash equilibrium for the game, with $x_{i}^{*}=1 / k$ for all $i \in V^{*}$ and $x_{i}^{*}=0$ for all $i \in V \backslash V^{*}$, where $k$ is the size of $V^{*}$.

Proof. Assume without loss of generality that $G^{*}=\left(V^{*}, E^{*}\right)$ with $V^{*}=\{1, \cdots, k\}$. Then, $A_{i, j}=0$ for all $i, j=1, \cdots, k, i \neq j$ and $A_{i, i}=1$ for all $i=1, \cdots, k$. Since $G^{*}$ supports $x^{*}, x_{i}^{*}>0$ for all $i \in V^{*}$ and $x_{i}^{*}=0$ for all $i \in V \backslash V^{*}$. Let $p_{i}\left(x^{*}\right)=\Sigma_{j} A_{i, j} x_{j}^{*}=\lambda^{*}$ for all $i \in V^{*}$. Then, $x_{i}^{*}=\lambda^{*}$ for all $i \in V^{*}$. Since $\Sigma_{i} x_{i}^{*}=1, \lambda^{*}=1 / k$. It follows that $x_{i}^{*}=\lambda^{*}$ with $x_{i}^{*}=1 / k$ for all $i \in V^{*}$. Since $G^{*}$ is a maximal independent set, for any $i \in V \backslash V^{*}$, there must be $l \in V^{*}$ such that $i$ and $l$ are connected, i.e., $(i, l) \in E$ and $A_{i, l}=1$. Therefore, for any $i \in V \backslash V^{*}$, $p_{i}\left(x^{*}\right)=\Sigma_{j} A_{i, j} x_{j}^{*} \geq x_{l}^{*}=\lambda^{*}$. By Theorem 2.1, $x^{*}$ is a Nash equilibrium.

Note that if $G^{*}$ is an independent set of $G$ but not a maximal one, there will be $i \in V \backslash V^{*}$ not connected with any $j \in V^{*}$ and $p_{i}\left(x^{*}\right)=\Sigma_{j} A_{i, j} x_{j}^{*}=0<\lambda^{*}$. Then, $x^{*}$ cannot be a Nash equilibrium. Theorem 2.2 suggests that if a maximal independent set of nodes is found in the network, an optimal distancing strategy can then be obtained for an individual by choosing only this set of nodes to visit or stay with an equal frequency $1 / k$ for each node, where $k$ is the size of the set. As a result, the population will then be distributed only on this set of nodes as well with an equally probable fraction $1 / k$ on each node. 


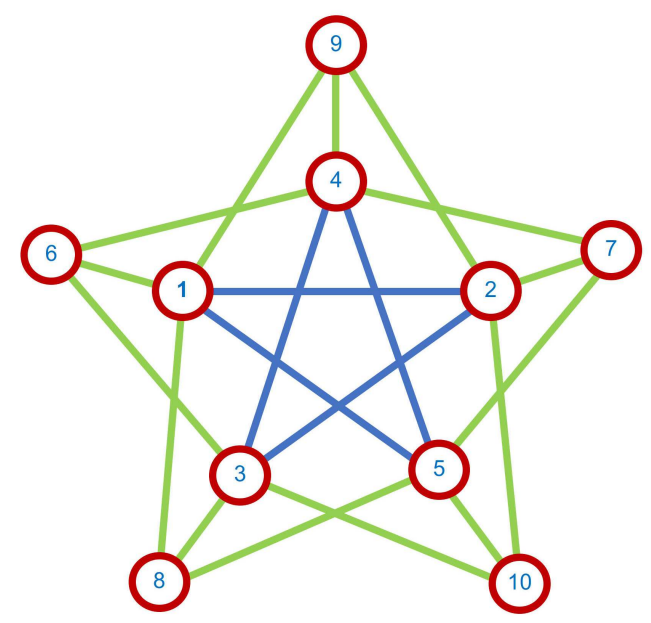

Figure 3: Social Distancing on Independent Sets. In the network shown in this figure, the network sites $\{3,5,9\}$ form a maximal independent set. An equilibrium strategy $x^{*}$ can be reached on this set of sites, with $x_{3}^{*}=x_{5}^{*}=x_{9}^{*}=1 / 3$ and all other elements $x_{j}^{*}=0$, and a minimal social contact $\lambda^{*}=1 / 3$. However, the sites $\{6,7,8,9,10\}$ form another maximal independent set. An equilibrium strategy $x^{*}$ can be reached on this set of sites, with $x_{6}^{*}=x_{7}^{*}=x_{8}^{*}=x_{9}^{*}=x_{10}^{*}=1 / 5$ and all other elements $x_{j}^{*}=0$, and a minimal social contact $\lambda^{*}=1 / 5$. The latter is an even better strategy than the former.

At equilibrium, every individual minimizes his/her total amount of social contacts to $\lambda^{*}=1 / k$. Therefore, the larger the maximal independent set, the better for minimizing the social contacts, and the strategy on a maximum independent set will be the best among all those supported by a maximal independent set.

Fig. 3 shows an example network, representing a world of 10 connected social sites. The inside 5 sites, $\{1,2,3,4,5\}$, may be town centers, well connected. The outside 5 sites, $\{6,7,8,9,10\}$, may be residential areas, each having access to some of the town centers. In this network, the sites $\{3,5,9\}$ form a maximal independent set. An equilibrium strategy $x^{*}$ can be reached on this set of sites, with $x_{3}^{*}=x_{5}^{*}=x_{9}^{*}=1 / 3$ and all other elements $x_{j}^{*}=0$, and a minimal social contact $\lambda^{*}=1 / 3$. However, the sites $\{6,7,8,9,10\}$ form another maximal independent set. An equilibrium strategy $x^{*}$ can be reached on this set of sites, with $x_{6}^{*}=x_{7}^{*}=x_{8}^{*}=x_{9}^{*}=x_{10}^{*}=1 / 5$ and all other elements $x_{j}^{*}=0$, and a minimal social contact $\lambda^{*}=1 / 5$. The latter is an even better strategy than the former.

An equilibrium strategy may as well be supported by a subnetwork where there are some connections among the nodes. A general class of subnetworks that may be selected by an equilibrium strategy is the regular networks. In a regular network, all nodes have the same number of connections with other nodes. In other words, all the nodes of a regular network have the same degree. If the degree is $r$, the network is called an $r$ regular network. Thus, a maximal independent set of a given network is a 0-regular network. A 1-regular network is where each node is connected only to another one; a 2-regular network is where each node is connected to two other nodes; and so on and so forth (see Fig. 2). 
A regular network can be connected or disconnected. In the latter case, it consists of a number of separated components, each being a connected regular network itself. For an $r$-regular subnetwork of $G$, the larger the subnetwork, the sparser the connectivity, and the better for social distancing. The largest possible $r$-regular subnetwork that is not contained in another $r$-regular subnetwork is called a maximal $r$-regular subnetwork. A distancing strategy on an $r$-regular subnetwork may not necessarily be an equilibrium strategy, but it always is if it is on a maximal $r$-regular subnetwork and if there is no less than $r+1$ connections to the subnetwork from an outside node.

Theorem 2.3. Let $x^{*} \in S$ be a strategy for the social distancing game on a network $G=(V, E)$. Let $G^{*}=\left(V^{*}, E^{*}\right)$ be the supporting subnetwork for $x^{*}$. Then, if $G^{*}$ is a maximal r-regular subnetwork and if there is no less than $r+1$ links to $G^{*}$ from a node $i \in V \backslash V^{*}, x^{*}$ is a Nash equilibrium for the game, with $x_{i}^{*}=1 / k$ for all $i \in V^{*}$ and $x_{i}^{*}=0$ for all $i \in V \backslash V^{*}$, where $k$ is the size of $V^{*}$.

Proof. Assume without loss of generality that $V^{*}=\{1, \cdots, k\}$. Then, $A^{*}=\left\{A_{i, j}, i, j=1, \cdots, k\right\}$ is the adjacency matrix of $G^{*}$, and each of its rows and columns has exactly $r+1$ elements equal to 1 . Since $G^{*}$ supports $x^{*}, x_{i}^{*}>0$ for all $i \in V^{*}$ and $x_{i}^{*}=0$ for all $i \in V \backslash V^{*}$. Then, $p_{i}\left(x^{*}\right)=\Sigma_{j} A_{i, j} x_{j}^{*}=\Sigma_{j \in V^{*}} A_{i, j} x_{j}^{*}$ for all $i \in V$. Let $p_{i}\left(x^{*}\right)=\Sigma_{j \in V^{*}} A_{i, j} x_{j}^{*}=\lambda^{*}$ for all $i \in V^{*}$. By adding all these equations, we obtain $r+1=k \lambda^{*}$, and $\lambda^{*}=(r+1) / k$. It follows that $\Sigma_{j \in V^{*}} A_{i, j} x_{j}^{*}=\lambda^{*}$ for all $i \in V^{*}$ with $x_{j}^{*}=1 / k$ for all $j \in V^{*}$. Since there is no less than $r+1$ links to $G^{*}$ from a node $i \in V \backslash V^{*}, p_{i}\left(x^{*}\right)=\Sigma_{j \in V^{*}} A_{i, j} x_{j}^{*} \geq(r+1) / k=\lambda^{*}$ for any $i \in V \backslash V^{*}$. By Theorem 2.1, $x^{*}$ is a Nash equilibrium.

Theorem 2.3 suggests that if a maximal $r$-regular subnetwork is found in the network, an optimal distancing strategy can then be obtained for an individual by choosing only this subnetwork to visit or stay with an equal frequency $1 / k$ for each node of the subnetwork, where $k$ is the number of the nodes in the subnetwork. As a result, the population will then be distributed only on this subnetwork as well with an equally probable fraction $1 / k$ on each node of the subnetwork. At equilibrium, every individual minimizes his/her total amount of social contacts to $\lambda^{*}=(r+1) / k$. Therefore, the larger the regular subnetwork or the smaller the $r$ value, the better for minimizing the social contacts.

Of particular interest among maximal $r$-regular subnetworks are those with $r>0$ and with a large number of separated components. For example, a maximal 1-regular subnetwork is just a set of separated 1-regular subnetworks of size 2; a maximal 2-regular subnetwork can be a large number of separated 2-regular subnetworks of size 3; etc. An equilibrium strategy on such a subnetwork means that the population can spread on a large number of independent locations, where at each location, there is a group of social sites with a low degree of connectivity. Such a strategy allows certain degrees of local contacts yet keeps maximal possible distances among individuals, and can therefore be considered as a more flexible strategy for social distancing than those on maximal independent sets.

Fig. 4 shows a network similar to the one in Fig. 3 except for some changes in contact connections. In this network, the sites $\{1,2,3,4,5\}$ form a 2-regular subnetwork. How- 


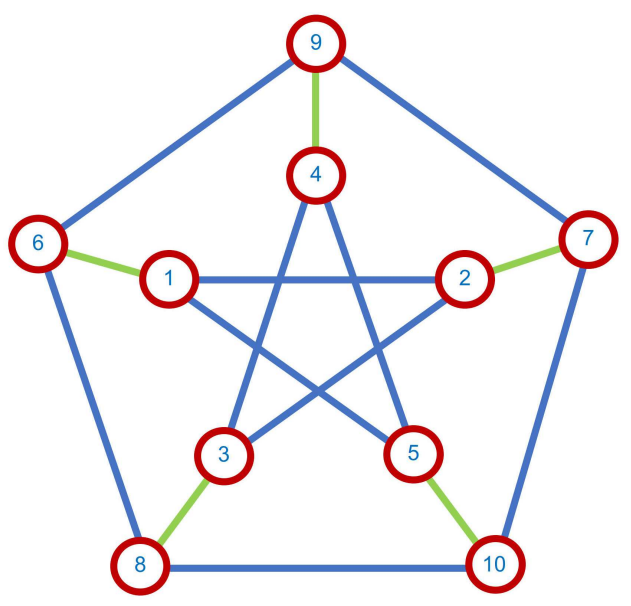

Figure 4: Social Distancing on Regular Subnetworks. In the network shown in this figure, the network sites $\{1,2,3,4,5\}$ form a 2-regular subnetwork. However, a strategy on this subnetwork may not be an equilibrium strategy because there are nodes outside of the subnetwork, say node 6 , with less than 3 connections to the nodes in the subnetwork. For the same reason, a strategy on the 2-regular subnetwork formed by the sites $\{6,7,8,9,10\}$ may not be an equilibrium strategy either. On the other hand, the network itself is a 3-regular network. An equilibrium strategy $x^{*}$ can be reached on the whole network with $x_{i}^{*}=1 / 10$ for $i=1, \cdots, 10$ and a minimal social contact $\lambda^{*}=2 / 5$.

ever, a strategy on this subnetwork may not be an equilibrium strategy because there are nodes outside the subnetwork, say node 6 , with less than 3 connections to the nodes in the subnetwork. For the same reason, a strategy on the 2-regular subnetwork formed by the sites $\{6,7,8,9,10\}$ may not be an equilibrium strategy, either. On the other hand, the whole network itself is a 3-regular network. An equilibrium strategy $x^{*}$ can be reached on the whole network with $x_{i}^{*}=1 / 10$ for all $i=1, \cdots, 10$, and a minimal social contact $\lambda^{*}=2 / 5$.

In the network in Fig. 4, there are also equilibrium strategies supported by other regular subnetworks. For example, the sites $\{1,2,4,8,9,10\}$ form a 1-regular subnetwork of size 6 . An equilibrium strategy $x^{*}$ can be reached on this subnetwork with $x_{1}^{*}=x_{2}^{*}=x_{4}^{*}=x_{8}^{*}=x_{9}^{*}=x_{10}^{*}=1 / 6$ and all other elements $x_{j}^{*}=0$, and a minimal social contact $\lambda^{*}=1 / 3$. There are total 5 such equilibrium strategies on this network. Furthermore, the sites $\{3,5,6,7\}$ form a maximal independent set of sites or in other words, a 0-regular subnetwork. An equilibrium strategy $x^{*}$ can be reached on this set of sites with $x_{3}^{*}=x_{5}^{*}=x_{6}^{*}=x_{7}^{*}=1 / 4$ and all other elements $x_{j}^{*}=0$, and a minimal social contact $\lambda^{*}=1 / 4$. There are total 4 such equilibrium strategies on this network. In terms of minimal social contact, among these strategies, the one on the maximal independent set, i.e., on a 0-regular subnetwork, is the best, because its social contact is the lowest. However, it is also the most strict strategy, for it does not allow any contacts across different sites. The strategy on the whole network, i.e., on a 3-regular network, has the highest social contact, but it is the most flexible strategy, allowing certain degrees of contacts across different sites. 


\section{Rigidity and flexibility of social distancing}

Once an equilibrium is reached, i.e., a distancing rule is established, can we make some small changes or adjustments on the strategy? This is a question of legitimate concern in practice, for we may not be able to commit to a rule all the time. The answer to the question is that it depends on whether the social contact is changed: if the contact is increased with small changes in the strategy, the rule needs to be enforced, and we say the strategy is rigid; if the contact is not changed, the rule is not strict, and we say the strategy is flexible; if the contact is decreased, the rule is broken, and we say the strategy is fragile. In order to further address this issue, we give a formal definition for rigidity, flexibility, and fragility for a distancing strategy in the following. We then analyze the strategies on maximal $r$-regular subnetworks with these properties.

Let $x^{*} \in S$ be an equilibrium strategy for the social distancing game on network $G$. Let $G^{*}$ be the subnetwork supporting $x^{*}$. Let $S^{*}=\left\{x \in S: x_{i}>0\right.$ if and only if $x_{i}^{*}>0$ for all $i=$ $1, \cdots, n\}$. Then, $S^{*}$ is the set of strategies all supported by $G^{*}$. We define rigidity, flexibility, and fragility of $x^{*}$ only for small changes of $x^{*}$ in $S^{*}$. Let $D^{*}=\left\{d \in R^{n}: d=x-x^{*}, x \in\right.$ $\left.S^{*}, x \neq x^{*}\right\}$. Then, $d \in D^{*}$ is a direction such that $x^{*}+\epsilon d$, a small change of $x^{*}$ along $d$, remains in $S^{*}$ for all $\epsilon>0$ sufficiently small.

Definition 3.1. Let $x^{*} \in S$ be an equilibrium strategy for the social distancing game on network $G$ supported by subnetwork $G^{*}$. Then, $x^{*}$ is said to be strongly rigid on $G^{*}$ if for any $d \in D^{*}$, $\pi\left(x^{*}+\epsilon d, x^{*}+\epsilon d\right)>\pi\left(x^{*}, x^{*}\right)$ for all $\epsilon>0$ sufficiently small.

Definition 3.2. Let $x^{*} \in S$ be an equilibrium strategy for the social distancing game on network $G$ supported by subnetwork $G^{*}$. Then, $x^{*}$ is said to be weakly rigid on $G^{*}$ if for any $d \in D^{*}$, $\pi\left(x^{*}+\epsilon d, x^{*}+\epsilon d\right) \geq \pi\left(x^{*}, x^{*}\right)$ for all $\epsilon>0$ sufficiently small.

Definition 3.3. Let $x^{*} \in S$ be an equilibrium strategy for the social distancing game on network $G$ supported by subnetwork $G^{*}$. Then, $x^{*}$ is said to be flexible on $G^{*}$ if there is $d \in D^{*}$ such that $\pi\left(x^{*}+\epsilon d, x^{*}+\epsilon d\right)=\pi\left(x^{*}, x^{*}\right)$ for all $\epsilon>0$ sufficiently small.

Definition 3.4. Let $x^{*} \in S$ be an equilibrium strategy for the social distancing game on network $G$ supported by subnetwork $G^{*}$. Then, $x^{*}$ is said to be fragile on $G^{*}$ if there is $d \in D^{*}$ such that $\pi\left(x^{*}+\epsilon d, x^{*}+\epsilon d\right)<\pi\left(x^{*}, x^{*}\right)$ for all $\epsilon>0$ sufficiently small.

Recall that $\pi(x, x)$ is the social contact of the population of strategy $x$. Therefore, the definition of rigidity basically implies that an equilibrium strategy $x^{*}$ is strongly (or weakly) rigid if it is a strict (or non-strict) local minimizer of the social contact $\pi(x, x)$ in $S^{*}$. In this sense, the strong (or weak) rigidity can be considered as a weaker version of strong (or weak) evolutionary stability for general evolutionary games [46,47]. Nonetheless, we call this property rigidity instead of stability because the corresponding strategy is considered as a rigid distancing rule in the context of social distancing. Note that based on the above definitions, if an equilibrium strategy is weakly (not strongly) rigid, it must 
be flexible; however, if it is flexible, it may not necessarily be weakly rigid; It could be fragile.

We now investigate these properties for strategies on maximal $r$-regular subnetworks. First, it is easy to justify that the equilibrium strategies on a maximal independent set are all strongly rigid: Small changes on such strategies always increase the amount of social contacts. In other words, there will be penalties for any changes on such strategies. On the other hand, the equilibrium strategies on any maximal $r$-regular subnetworks with $r>0$ are always flexible: In this case, we can find some connected nodes in the subnetworks. By increasing the frequencies on some of these nodes while decreasing on others, we can make some small changes on the strategy without changing the original amount of social contacts. Of these flexible strategies, some may be weakly rigid, but others may be fragile.

Theorem 3.1. Let $x^{*} \in S$ be an equilibrium strategy for the social distancing game on network $G=(V, E)$. If $x^{*}$ is supported by a maximal independent set $G^{*}=\left(V^{*}, E^{*}\right)$, then $x^{*}$ is strongly rigid on $G^{*}$.

Proof. Let $x^{*}+\epsilon d$ be a change from $x^{*}$ for any $d \in D^{*}$ and $\epsilon>0$ sufficiently small. Then,

$$
\begin{aligned}
\pi\left(x^{*}+\epsilon d, x^{*}+\epsilon d\right) & =\left(x^{*}+\epsilon d\right)^{T} A\left(x^{*}+\epsilon d\right) \\
& =x^{* T} A x^{*}+2 \epsilon d^{T} A x^{*}+\epsilon^{2} d^{T} A d .
\end{aligned}
$$

Note that $d=x-x^{*}$ for some $x \in S^{*}, x \neq x^{*}$. Therefore, $d \neq 0$, but $d_{i}=0$ for $i \in V \backslash V^{*}$, and $\Sigma_{i \in V^{*}} d_{i}=0$. Note also that $\left(A x^{*}\right)_{i}=\lambda^{*}$ for all $i \in V^{*}$ and therefore, $d^{T} A x^{*}=\Sigma_{i \in V^{*}} d_{i}\left(A x^{*}\right)_{i}=$ 0 . In addition, since $G^{*}$ is a maximal independent set, $\left\{A_{i, j}, i, j \in V^{*}\right\}$, the adjacency matrix of $G^{*}$, is an identity matrix. Therefore, $d^{T} A d=\Sigma_{i, j \in V^{*}} d_{i} A_{i, j} d_{j}=\Sigma_{i \in V^{*}} d_{i}^{2}>0$. It follows that

$$
\begin{aligned}
\pi\left(x^{*}+\epsilon d, x^{*}+\epsilon d\right) & =x^{* T} A x^{*}+\epsilon^{2} d^{T} A d \\
& >x^{* T} A x^{*}=\pi\left(x^{*}, x^{*}\right) .
\end{aligned}
$$

By Definition 3.1, $x^{*}$ is strongly rigid on $G^{*}$.

Theorem 3.2. Let $x^{*} \in S$ be an equilibrium strategy for the social distancing game on network $G=(V, E)$. If $x^{*}$ is supported by a maximal $r$-regular subnetwork $G^{*}=\left(V^{*}, E^{*}\right)$ with $r>0$, then $x^{*}$ is flexible on $G^{*}$.

Proof. Since $G^{*}$ is an $r$-regular subnetwork with $r>0$, there must be $i, j \in V^{*}$ such that $(i, j) \in E^{*}$ and $A_{i, j}=1$. Let $d$ be a vector such that $d_{i}=-d_{j}=\delta$ with $0<\delta<\min \left\{1-x_{i}^{*}, x_{j}^{*}\right\}$ and $d_{l}=0$ for all $l \neq i, j$. Then, $d \in D^{*}$. Let $x^{*}+\epsilon d$ be a change from $x^{*}$ along $d$ for $\epsilon>0$ sufficiently small. Then,

$$
\begin{aligned}
\pi\left(x^{*}+\epsilon d, x^{*}+\epsilon d\right) & =\left(x^{*}+\epsilon d\right)^{T} A\left(x^{*}+\epsilon d\right) \\
& =x^{* T} A x^{*}+2 \epsilon d^{T} A x^{*}+\epsilon^{2} d^{T} A d .
\end{aligned}
$$


Since $\left(A x^{*}\right)_{k}=\lambda^{*}$ for all $k \in V^{*}, d^{T} A x^{*}=\left(d_{i}+d_{j}\right) \lambda^{*}=0$. Also, $d^{T} A d=A_{i, i} d_{i}^{2}+2 A_{i, j} d_{i} d_{j}+$ $A_{j, j} d_{j}^{2}=0$. It follows that

$$
\begin{aligned}
\pi\left(x^{*}+\epsilon d, x^{*}+\epsilon d\right) & =\left(x^{*}+\epsilon d\right)^{T} A\left(x^{*}+\epsilon d\right) \\
& =x^{* T} A x^{*}=\pi\left(x^{*}, x^{*}\right) .
\end{aligned}
$$

By Definition 3.3, $x^{*}$ is flexible on $G^{*}$.

Based on Theorems 3.1 and 3.2, for the example distancing problem shown in Fig. 4, the equilibrium strategy on a maximal independent set such as $\{3,5,6,7\}$ is strongly rigid, and the one on a maximal 1-regular subnetwork such as $\{1,2,4,8,9,10\}$ is flexible. The equilibrium strategy on the whole network, which is a 3-regular network, is also flexible. Therefore, as described in the proof for Theorem 3.2, for this strategy, the frequencies can be exchanged between any two connected nodes, say nodes 1 and 2, by moving a small amount from one node to the other. For example, by moving $1 / 20$ from node 1 to node 2 , the frequency on node 1 decreases to $1 / 20$ from $1 / 10$ while on node 2 increases to $3 / 20$ from $1 / 10$, yet the minimal social contact for every individual remains to be $2 / 5$.

Note that an $r$-regular network must have at least $r+1$ nodes. We call an $r$-regular network of size $r+1$ a minimal $r$-regular network. A minimal $r$-regular network must be a complete network, i.e., each node is connected with all others in the network. It turns out that an equilibrium strategy for the social distancing game on a network is always weakly rigid if it is supported by a maximal $r$-regular subnetwork whose components are all minimal $r$-regular subnetworks with $r>0$ :

Theorem 3.3. Let $x^{*} \in S$ be an equilibrium strategy for the social distancing game on network $G=(V, E)$. Then, $x^{*}$ is weakly rigid if it is supported by a maximal $r$-regular subnetwork $G^{*}=$ $\left(V^{*}, E^{*}\right)$ whose components are all minimal r-regular subnetworks with $r>0$.

Proof. Let $G_{i}^{*}=\left(V_{i}^{*}, E_{i}^{*}\right)$ be the $i$ th component of $G^{*}$ and $A^{(i)}$ the adjacency matrix of $G_{i}^{*}$, $i=1, \cdots, m$. Then, $A^{(i)}$ is a matrix of all $1^{\prime}$ s. Let $x^{*}+\epsilon d$ be a change from $x^{*}$ for any $d \in D^{*}$ and $\epsilon>0$ sufficiently small.

$$
\begin{aligned}
\pi\left(x^{*}+\epsilon d, x^{*}+\epsilon d\right) & =\left(x^{*}+\epsilon d\right)^{T} A\left(x^{*}+\epsilon d\right) \\
& =x^{* T} A x^{*}+2 \epsilon d^{T} A x^{*}+\epsilon^{2} d^{T} A d .
\end{aligned}
$$

Note that $d=x-x^{*}$ for some $x \in S^{*}, x \neq x^{*}$. Therefore, $d \neq 0$, but $d_{i}=0$ for $i \in V \backslash V^{*}$, and $\Sigma_{i \in V^{*}} d_{i}=0$. Note also that $\left(A x^{*}\right)_{i}=\lambda^{*}$ for all $i \in V^{*}$ and therefore, $d^{T} A x^{*}=\Sigma_{i \in V^{*}} d_{i}\left(A x^{*}\right)_{i}=$ 0 . Let $c_{i}=\left\{d_{j}: j \in V_{i}^{*}\right\}$. Then,

$$
d^{T} A d=\Sigma_{i=1: m} c_{i}^{T} A^{(i)} c_{i}=\Sigma_{i=1: m}\left(\Sigma_{j \in V_{i}^{*}} d_{j}\right)^{2} \geq 0
$$

It follows that

$$
\begin{aligned}
\pi\left(x^{*}+\epsilon d, x^{*}+\epsilon d\right) & =\left(x^{*}+\epsilon d\right)^{T} A\left(x^{*}+\epsilon d\right) \\
& =x^{* T} A x^{*}+\epsilon^{2} d^{T} A d \geq x^{* T} A x^{*}=\pi\left(x^{*}, x^{*}\right) .
\end{aligned}
$$

By Definition $3.2, x^{*}$ is weakly rigid on $G^{*}$. 


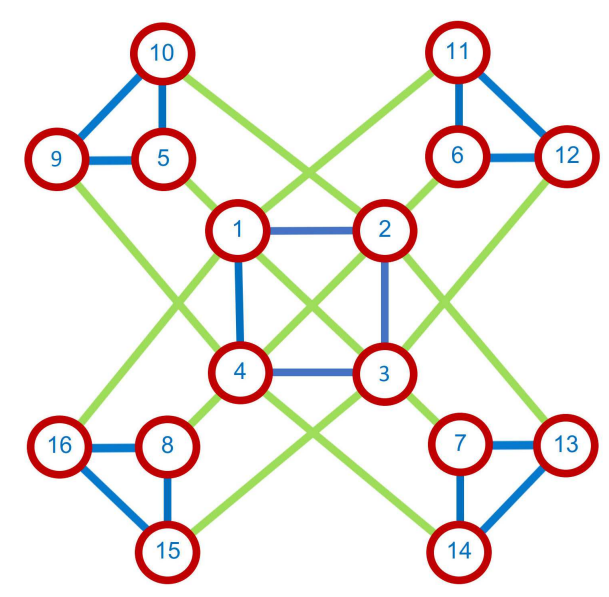

Figure 5: Rigidity and Flexibility of Distancing Strategies. The social distancing game on this network has at least three equilibrium strategies: one on the maximal 0-regular subnetwork or in other words, the maximal independent set $\{5,6,7,8\}$ with the minimal social contact equal to $1 / 4$; one on the maximal 1-regular subnetwork $\{9,10,11,12,13,14,15,16\}$ with the minimal social contact equal to $1 / 4$; and one on the maximal 2-regular subnetwork $\{5,6,7,8,9,10,11,12,13,14,15,16\}$ with the minimal social contact equal to $1 / 4$. The first one is strongly rigid. The second and third are flexible but weakly rigid.

From the above proof, we see that if we update $x^{*}$ with a vector $d$ such that $\Sigma_{j \in V_{i}^{*}} d_{j}=0$ for all $i=1, \cdots, m$, we will have $d^{T} A d=0$, and hence $\left(x^{*}+\epsilon d\right)^{T} A\left(x^{*}+\epsilon d\right)=x^{* T} A x^{*}$, i.e., the minimal amount of social contacts will remain to be the same. This means that we can make such small changes in frequencies on the subnetwork components independently without changing the original amount of social contacts across the network. In fact, we can make such changes on any group of supporting components as long as they are minimal regular subnetworks.

Fig. 5 shows an example network with 16 social sites. It is structured like a small town, with 4 connected town centers in the middle and 4 separated residential areas outside around. Every residential area consists of 3 well-connected activity sites, each having access to one of the town centers. The social distancing game on this network has at least three equilibrium strategies: one on the maximal 0-regular subnetwork or in other words, the maximal independent set $\{5,6,7,8\}$ with the minimal social contact equal to $1 / 4$; one on the maximal 1-regular subnetwork $\{9,10,11,12,13,14,15,16\}$ with the minimal social contact equal to $1 / 4$; and one on the maximal 2-regular subnetwork $\{5,6,7,8,9,10,11,12,13,14,15,16\}$ with the minimal social contact equal to $1 / 4$. The minimal social contacts of the three strategies are all the same. However, based on Theorems 3.1, 3.2, and 3.3, the first one is strongly rigid; the second and third are flexible but weakly rigid. In addition, the supporting subnetworks for the second and third strategies consist of separated minimal regular subnetworks. The frequencies on those minimal subnetworks can be adjusted independently without affecting the social contacts of the individuals. For example, for the third strategy, for any minimal 2-regular subnetwork component, say the subnetwork $\{5,9,10\}$, a small fraction $1 / 24$ can be moved from 
each of the outer most two sites, say the sites 9 and 10, to the inner site, say the site 5 . The fractions on the outer sites are then decreased from $1 / 12$ to $1 / 24$ while on the inner site is increased from $1 / 12$ to $4 / 24$. However, such changes will not affect the original minimized social contact of the population.

Finally, if a maximal $r$-regular subnetwork is connected but not minimal, or disconnected but at least one of the components is not minimal, then an equilibrium strategy supported by such a subnetwork will not be weakly rigid though still flexible. It will in fact be fragile, i.e., there will be some way to update the strategy on the subnetwork to decrease the social contacts among the individuals. Such changes will make the population to break away from the current equilibrium state. An example fragile strategy is the one on the whole network for the game shown in Fig. 4. The whole network is a 3-regular network, but it is not minimal.

Theorem 3.4. Let $x^{*} \in S$ be an equilibrium strategy for the social distancing game on a network $G=(V, E)$ supported by a maximal $r$-regular subnetwork $G^{*}=\left(V^{*}, E^{*}\right), r>0$. If $G^{*}$ is connected but not minimal, or disconnected but at least one of its components is not minimal, then $x^{*}$ is fragile on $G^{*}$.

Proof. Without loss of generality, we consider only the case where $G^{*}$ is connected. If $G^{*}$ is not a minimal $r$-regular subnetwork, there must be three nodes $i, j, l$ such that $(i, l),(j, l) \in$ $E^{*}$, but $(i, j) \notin E^{*}$. Let $d$ be a vector such that $d_{k}=0$ for all $k \neq i, j, l, d_{i}=d_{j}=\delta$, with $0<\delta<\min \left\{1-x_{i}^{*}, 1-x_{j}^{*}, x_{l}^{*} / 2\right\}$, and $d_{i}+d_{j}+d_{l}=0$. Then, $d \in D^{*}$. Let $x^{*}+\epsilon d$ be a change from $x^{*}$ along $d \in D^{*}$ for $\epsilon>0$ sufficiently small. Then,

$$
\begin{aligned}
\pi\left(x^{*}+\epsilon d, x^{*}+\epsilon d\right) & =\left(x^{*}+\epsilon d\right)^{T} A\left(x^{*}+\epsilon d\right) \\
& =x^{* T} A x^{*}+2 \epsilon d^{T} A x^{*}+\epsilon^{2} d^{T} A d .
\end{aligned}
$$

Note that $\left(A x^{*}\right)_{k}=\lambda^{*}$ for all $k \in V^{*}$, and therefore,

$$
d^{T} A x^{*}=\lambda^{*} \Sigma_{k \in V^{*}} d_{k}=d_{i}+d_{j}+d_{l}=0 .
$$

Note also,

$$
\begin{aligned}
d^{T} A d & =d_{i}^{2}+d_{j}^{2}+d_{l}^{2}+2 d_{i} d_{l}+2 d_{j} d_{l} \\
& =\left(d_{i}+d_{j}+d_{l}\right)^{2}-2 d_{i} d_{j}=-2 d_{i} d_{j}<0
\end{aligned}
$$

Therefore,

$$
\begin{aligned}
\pi\left(x^{*}+\epsilon d, x^{*}+\epsilon d\right) & =\left(x^{*}+\epsilon d\right)^{T} A\left(x^{*}+\epsilon d\right) \\
& =x^{* T} A x^{*}+\epsilon^{2} d^{T} A d \\
& <x^{* T} A x^{*}=\pi\left(x^{*}, x^{*}\right) .
\end{aligned}
$$

By Definition 3.4, $x^{*}$ is fragile on $G^{*}$. 


\section{Extension to weighted networks}

So far, in all our discussions, we have assumed that the social sites are all the same for making social contacts. However, in real situation, different sites may have different sizes, different population densities, different activities, and hence different contact rates. Therefore, a more realistic model for a given world of social sites would be a weighted network of social sites, with each site assigned a contact value. The total amount of social contacts an individual can make will then be those made within each site and through connected sites, all weighted by the contact values of the sites. We show that for such a weighted network of social sites, we can define a similar social distancing game as we have discussed in previous sections, and have similar distancing strategies and related rigidity and flexibility properties.

Let $A$ be the adjacency matrix of a given network $G$. Suppose for each social site $i$, we assign a contact value $w_{i} \geq 1$. Then, in a population of strategy $y$, the total amount of social contacts an individual can make at social site $i$ will be $p_{i}(y)=\Sigma_{j} \tilde{A}_{i, j} y_{j}$, where $\tilde{A}_{i, j}$ is a value dependent of $w_{i}$ and $w_{j}$. We consider two methods to define $\tilde{A}_{i, j}$ : (a) with an additive weight, $\tilde{A}_{i, j}=\left(w_{i} A_{i, j}+A_{i, j} w_{j}\right) / 2$, i.e., if $A_{i, j}=1, \tilde{A}_{i, j}$ is the average of the weights of sites $i$ and $j$; and (b) with a multiplicative weight, $\tilde{A}_{i, j}=w_{i} A_{i, j} w_{j}$, i.e., if $A_{i, j}=1, \tilde{A}_{i, j}$ is the multiplication of the weights of sites $i$ and $j$. Then, if an individual takes a strategy $x$ to visit all the social sites, the total amount of social contacts the individual can make will be $\Sigma_{i} x_{i} p_{i}(y)=\Sigma_{i} \Sigma_{j} x_{i} \tilde{A}_{i, j} y_{j}=x^{T} \tilde{A} y$, where $\tilde{A}=\left\{\tilde{A}_{i, j}\right\}$. Let $w=\left(w_{1}, \cdots, w_{n}\right)^{T}$, and $W=\operatorname{diag}[w]$, i.e., the diagonal matrix with $w$ as its diagonal vector. Then, $\tilde{A}$ is a matrix with the same nonzero entries of $A$, but weighted by $W$, and with additive weights, $\tilde{A}=(W A+A W) / 2$, and with multiplicative weights, $\tilde{A}=W A W$.

By considering $\tilde{A}$ as a weighted adjacency matrix of network $G$, and $\pi(x, y)=x^{T} \tilde{A} y$ as a payoff function, we can then define a social distancing game, where each individual tries to minimize his/her weighted social contacts $\pi(x, y)=x^{T} \tilde{A} y$. A Nash equilibrium of this game is then a strategy $x^{*}$ such that

$$
\pi\left(x^{*}, x^{*}\right) \leq \pi\left(x, x^{*}\right), \quad \forall x \in S .
$$

We call this game a weighted social distancing game on network G. Recall for social distancing, we count the contacts among individuals in the same sites, and therefore $A_{i, i}=1$ for all $i=1, \cdots, n$. It follows that $\tilde{A}_{i, i}$ are nonzero, and $\tilde{A}_{i, i}=w_{i}$ for additive weights, and $\tilde{A}_{i, i}=w_{i}^{2}$ for multiplicative weights for all $i=1, \cdots, n$.

The conditions for a given strategy to be a Nash equilibrium for the above weighted social distancing game are the same as those for the social distancing game on an unweighted network as given in Theorem 2.1, except that the adjacency matrix $A$ is replaced by the weighted adjacency matrix $\tilde{A}$. We state these conditions in the following theorem as a reference without repeating the proof.

Theorem 4.1. Let $\tilde{A}$ be the weighted adjacency matrix of network $G$. Then, a strategy $x^{*} \in S$ is a Nash equilibrium of the weighted social distancing game on $G$ if and only if there is a scalar $\lambda^{*}$ 
such that $p_{i}\left(x^{*}\right)=\lambda^{*}$ for all $i$ such that $x_{i}^{*}>0$ and $p_{i}\left(x^{*}\right) \geq \lambda^{*}$ for all $i$ such that $x_{i}^{*}=0$, where $p_{i}\left(x^{*}\right)=\Sigma_{j} \tilde{A}_{i, j} x_{j}^{*}$.

Note that the first set of conditions, which we call the equality conditions, requires the social contacts on all selected nodes to be equal; and the second set, which we call the inequality conditions, requires the social contacts on the unselected nodes to be no less than those on the selected ones; and in either case, the social contacts are weighted by the weights on the corresponding network sites.

In a weighted network, an optimal distancing strategy can also be obtained by distancing on a maximal independent set of social sites, i.e., with a maximal independent set as the supporting subnetwork. However, the distribution of the frequencies over the selected sites is not necessarily uniform any more, for the social sites are now weighted by the contact values. Also, the inequality conditions for the strategy are not necessarily satisfied automatically, either:

Theorem 4.2. Let $x^{*} \in S$ be a strategy for the weighted social distancing game on $G=(V, E)$, with $\tilde{A}=(A W+W A) / 2$. Let $G^{*}=\left(V^{*}, E^{*}\right)$ be the supporting subnetwork for $x^{*}$. Then, if $G^{*}$ is a maximal independent set of $G$ and if for any $i \in V \backslash V^{*}$, there is $j \in V^{*}$ such that $(i, j) \in E$ and $w_{i} \geq w_{j}, x^{*}$ is a Nash equilibrium for the game, with $x_{i}^{*}=\bar{w} / w_{i}$ for all $i \in V^{*}$ and $x_{i}^{*}=0$ for all $i \in V \backslash V^{*}$, where $1 / \bar{w}=\Sigma_{i \in V^{*}} 1 / w_{i}$.

Proof. Since $G^{*}$ supports $x^{*}, x_{i}^{*}>0$ for all $i \in V^{*}$ and $x_{i}^{*}=0$ for all $i \in V \backslash V^{*}$. Also, $\tilde{A}_{i, i}=w_{i}$ for all $i \in V^{*}$, and $\tilde{A}_{i, j}=0$ for all $i, j \in V^{*}, i \neq j$. Let $p_{i}\left(x^{*}\right)=\Sigma_{j \in V^{*}} \tilde{A}_{i, j} x_{j}^{*}=\lambda^{*}$ for all $i \in V^{*}$. Then, $\tilde{A}_{i, i} x_{i}^{*}=\lambda^{*}$, i.e., $w_{i} x_{i}^{*}=\lambda^{*}$, for all $i \in V^{*}$. Since $\Sigma_{i \in V^{*}} x_{i}^{*}=1, \lambda^{*}=\bar{w}$. It follows that $w_{i} x_{i}^{*}=\lambda^{*}$ with $x_{i}^{*}=\bar{w} / w_{i}$ for all $i \in V^{*}$. Since for any $i \in V \backslash V^{*}$, there is $l \in V^{*}$ such that $(i, l) \in E$ and $w_{i} \geq w_{l}, \tilde{A}_{i, l}=\left(w_{i}+w_{l}\right) / 2 \geq w_{l}$. Therefore, for any $i \in V \backslash V^{*}$,

$$
\begin{aligned}
p_{i}\left(x^{*}\right) & =\Sigma_{j \in V^{*}} \tilde{A}_{i, j} x_{j}^{*} \\
& =\Sigma_{j \in V^{*},(i, j) \in E}\left(w_{i}+w_{j}\right) x_{j}^{*} / 2 \\
& \geq\left(w_{i}+w_{l}\right) x_{l}^{*} / 2 \geq w_{l} x_{l}^{*} \\
& =\bar{w}=\lambda^{*} .
\end{aligned}
$$

By Theorem 4.1, $x^{*}$ is a Nash equilibrium.

Theorem 4.3. Let $x^{*} \in S$ be a strategy for the weighted social distancing game on $G=(V, E)$, with $\tilde{A}=W A W$. Let $G^{*}=\left(V^{*}, E^{*}\right)$ be the supporting subnetwork for $x^{*}$. Then, if $G^{*}$ is a maximal independent set of $G$ and if for any $i \in V \backslash V^{*}$, there is $j \in V^{*}$ such that $(i, j) \in E$ and $w_{i} \geq w_{j}, x^{*}$ is a Nash equilibrium for the game, with $x_{i}^{*}=\tilde{w}^{2} / w_{i}^{2}$ for all $i \in V^{*}$ and $x_{i}^{*}=0$ for all $i \in V \backslash V^{*}$, where $1 / \tilde{w}^{2}=\Sigma_{i \in V^{*}} 1 / w_{i}^{2}$.

Proof. Since $G^{*}$ supports $x^{*}, x_{i}^{*}>0$ for all $i \in V^{*}$ and $x_{i}^{*}=0$ for all $i \in V \backslash V^{*}$. Also, $\tilde{A}_{i, i}=w_{i}^{2}$ for all $i \in V^{*}$, and $\tilde{A}_{i, j}=0$ for all $i, j \in V^{*}, i \neq j$. Let $p_{i}\left(x^{*}\right)=\Sigma_{j \in V^{*}} \tilde{A}_{i, j} x_{j}^{*}=\lambda^{*}$ for all $i \in V^{*}$. Then, $\tilde{A}_{i, i} x_{i}^{*}=\lambda^{*}$, i.e., $w_{i}^{2} x_{i}^{*}=\lambda^{*}$, for all $i \in V^{*}$. Since $\Sigma_{i \in V^{*}} x_{i}^{*}=1, \lambda^{*}=\tilde{w}^{2}$. It follows that 
$w_{i}^{2} x_{i}^{*}=\lambda^{*}$ with $x_{i}^{*}=\tilde{w}^{2} / w_{i}^{2}$ for all $i \in V^{*}$. Since for any $i \in V \backslash V^{*}$, there is $l \in V^{*}$ such that $(i, l) \in E$ and $w_{i} \geq w_{l}, \tilde{A}_{i, l}=w_{i} w_{l} \geq w_{l}^{2}$. Therefore, for any $i \in V \backslash V^{*}$,

$$
\begin{aligned}
p_{i}\left(x^{*}\right) & =\Sigma_{j \in V^{*}} \tilde{A}_{i, j} x_{j}^{*} \\
& =\Sigma_{j \in V^{*},(i, j) \in E} w_{i} w_{j} x_{j}^{*} \\
& \geq w_{i} w_{l} x_{l}^{*} \geq w_{l}^{2} x_{l}^{*} \\
& =\tilde{w}^{2}=\lambda^{*} .
\end{aligned}
$$

By Theorem 4.1, $x^{*}$ is a Nash equilibrium.

Note that the above two theorems show that an optimal strategy $x^{*}$ supported by a maximal independent set has $x_{i}^{*}=\bar{w} / w_{i}$ for networks with additive weights and $x_{i}^{*}=$ $\tilde{w}^{2} / w_{i}^{2}$ for networks with multiplicative weights. In either case, $x_{i}^{*}$, the frequency of visiting or staying at social site $i$, is inversely proportional to $w_{i}$ or $w_{i}^{2}$, i.e., the contact value of social site $i$ or its square. This implies that the larger the contact value at a given social site, the smaller the corresponding visiting frequency and hence the probable fraction of the population at the site. In addition, the total amount of social contacts every individual can make at equilibrium is given by the parameter $\lambda^{*}$, which is equal to $\bar{w}$ for networks with additive weights and to $\tilde{w}^{2}$ for networks with multiplicative weights. In either case, the larger the number of nodes in the supporting subnetwork or the lighter the assigned weights, the smaller the $\lambda^{*}$ value, i.e., the minimal amount of social contacts at equilibrium.

As an example, consider the network in Fig. 4 . Assume that the nodes in $\{1,2,3,4,5\}$ are all assigned a weight 2, i.e., $w_{1}=w_{2}=w_{3}=w_{4}=w_{5}=2$, and the nodes in $\{6,7,8,9,10\}$ are all assigned a weight 1 , i.e., $w_{6}=w_{7}=w_{8}=w_{9}=w_{10}=1$. Consider three maximal independent sets of the network, $\{3,5,9\},\{4,6,7\}$, and $\{3,5,6,7\}$. Assume that the additive weights are used for the calculation of the social contacts. First, for $\{3,5,9\}, \lambda^{*}=\bar{w}=$ $1 /\left(1 / w_{3}+1 / w_{5}+1 / w_{9}\right)=1 /(1 / 2+1 / 2+1)=1 / 2$. Let $x_{3}^{*}=\bar{w} / w_{3}=1 / 4, x_{5}^{*}=\bar{w} / w_{5}=1 / 4$, $x_{9}^{*}=\bar{w} / w_{9}=2 / 4$, and $x_{i}^{*}=0$ for all $i \neq 3,5,9$. Then, $x^{*}$ satisfies the equality conditions in Theorem 4.1. However, for $i=8, p_{i}\left(x^{*}\right)=\left(w_{8}+w_{3}\right) x_{3}^{*} / 2=3 / 8<1 / 2=\lambda^{*}$. Then, $x^{*}$ violates the inequality conditions in Theorem 4.1 , and therefore cannot be a Nash equilibrium. Second, for $\{4,6,7\}, \lambda^{*}=\bar{w}=1 /\left(1 / w_{4}+1 / w_{6}+1 / w_{7}\right)=1 /(1 / 2+1+1)=2 / 5$. Let $x_{4}^{*}=\bar{w} / w_{4}=1 / 5, x_{6}^{*}=\bar{w} / w_{6}=2 / 5, x_{7}^{*}=\bar{w} / w_{7}=2 / 5$, and $x_{i}^{*}=0$ for $i \neq 4,6,7$. Also, for any $i \neq 4,6,7$, there is a node $l \in\{4,6,7\}$ such that $i$ and $l$ are connected and $w_{i} \geq w_{l}$. Therefore, by Theorem $4.2, x^{*}$ is a Nash equilibrium, with the total amount of social contacts minimized to $\lambda^{*}=2 / 5$. Third, for $\{3,5,6,7\}, \lambda^{*}=\bar{w}=1 /\left(1 / w_{3}+w_{5}+1 / w_{6}+1 / w_{7}\right)=$ $1 /(1 / 2+1 / 2+1+1)=1 / 3$. Let $x_{3}^{*}=\bar{w} / w_{3}=1 / 6, x_{5}^{*}=\bar{w} / w_{5}=1 / 6, x_{6}^{*}=\bar{w} / w_{6}=2 / 6$, $x_{7}^{*}=\bar{w} / w_{7}=2 / 6$, and $x_{i}^{*}=0$ for $i \neq 3,5,6,7$. Also, for any $i \neq 3,5,6,7$, there is a node $l \in\{3,5,6,7\}$ such that $i$ and $l$ are connected and $w_{i} \geq w_{l}$. Therefore, by Theorem $4.2, x^{*}$ is a Nash equilibrium, with the total amount of social contacts minimized to $\lambda^{*}=1 / 3$.

The strategies on a general maximal $r$-regular subnetwork are more complicated if the subnetwork has arbitrary weights assigned. However, they can still be determined 
easily, if the weights are the same for all the nodes in each component of the subnetwork. For the following two theorems, we therefore assume that the supporting subnetwork $G^{*}$ is a maximal $r$-regular subnetwork and consists of $m$ separated components $G_{1}^{*}, \cdots, G_{m}^{*}$. For each component $G_{k}^{*}$, a weight $w_{k}^{\prime}$ is assigned to all its nodes.

Theorem 4.4. Let $x^{*} \in S$ be a strategy for the weighted social distancing game on $G=(V, E)$, with $\tilde{A}=(A W+W A) / 2$. Let $G^{*}=\left(V^{*}, E^{*}\right)$ be the supporting subnetwork for $x^{*}$. Assume that $G^{*}$ consists of $m$ separated components $G_{k}^{*}=\left(V_{k}^{*}, E_{k}^{*}\right)$, each assigned a weight $w_{k}^{\prime}, k=1, \cdots, m$. Then, if $G^{*}$ is a maximal $r$-regular subnetwork and if for any node $i \in V \backslash V^{*}$, there are at least $r+1$ nodes $j \in V^{*}$ such that $(i, j) \in E$ and $w_{i} \geq w_{j}, x^{*}$ is a Nash equilibrium for the game, with $x_{i}^{*}=\bar{w} / w_{k}^{\prime}$ for all $i \in V_{k}^{*}, k=1, \cdots, m$, and $x_{i}^{*}=0$ for all $i \in V \backslash V^{*}$, where $1 / \bar{w}=\Sigma_{k} m_{k} / w_{k}^{\prime}$, with $m_{k}$ being the size of $V_{k}^{*}$.

Proof. Since $G^{*}$ supports $x^{*}, x_{i}^{*}>0$ for all $i \in V^{*}$ and $x_{i}^{*}=0$ for all $i \in V \backslash V^{*}$. Then $p_{i}\left(x^{*}\right)=$ $\Sigma_{j} \tilde{A}_{i, j} x_{j}^{*}=\Sigma_{j \in V_{k}^{*}} \tilde{A}_{i, j} x_{j}^{*}=w_{k}^{\prime} \Sigma_{(i, j) \in E_{k}^{*}} x_{j}^{*}$ for all $i \in V_{k}^{*}$. Let $w_{k}^{\prime} \sum_{(i, j) \in E_{k}^{*}} x_{j}^{*}=\lambda^{*}$ for all $i \in V_{k}^{*}$. By adding the equations for all $i \in V_{k}^{*}$, we obtain $w_{k}^{\prime}(r+1) \Sigma_{j \in V_{k}^{*}} x_{j}^{*}=m_{k} \lambda^{*}$. By adding the latter equations for all $k$, we then obtain $\lambda^{*}=(r+1) \bar{w}$. It follows that $w_{k}^{\prime} \sum_{(i, j) \in E_{k}^{*}} x_{j}^{*}=\lambda^{*}$ for all $i \in V_{k}^{*}$ with $x_{j}^{*}=\bar{w} / w_{k}^{\prime}$ for all $j \in V_{k}^{*}$. Note that for any node $i \in V \backslash V^{*}$,

$$
\begin{aligned}
p_{i}\left(x^{*}\right) & =\Sigma_{j} \tilde{A}_{i, j} x_{j}^{*}=\Sigma_{k} \Sigma_{j \in V_{k}^{*}} \tilde{A}_{i, j} x_{j}^{*} \\
& =\Sigma_{k} \Sigma_{j \in V_{k}^{*},(i, j) \in E}\left(w_{i}+w_{j}\right) x_{j}^{*} / 2 .
\end{aligned}
$$

Since there are at least $r+1$ nodes $l \in V^{*}$ such that $(i, l) \in E$ and $w_{i} \geq w_{l}$,

$$
\begin{aligned}
p_{i}\left(x^{*}\right) & \geq \Sigma_{k} \Sigma_{l \in V_{k}^{*},(i, l) \in E}\left(w_{i}+w_{l}\right) x_{l}^{*} / 2 \\
& \geq \Sigma_{k} \Sigma_{l \in V_{k}^{*},(i, l) \in E} w_{l} x_{l}^{*} \\
& =\Sigma_{k} \Sigma_{l \in V_{k}^{*},(i, l) \in E} \bar{w} \\
& \geq(r+1) \bar{w}=\lambda^{*} .
\end{aligned}
$$

By Theorem $4.1, x^{*}$ is a Nash equilibrium.

Theorem 4.5. Let $x^{*} \in S$ be a strategy for the weighted social distancing game on $G=(V, E)$, with $\tilde{A}=W A W$. Let $G^{*}=\left(V^{*}, E^{*}\right)$ be the supporting subnetwork for $x^{*}$. Assume that $G^{*}$ consists of $m$ separated components $G_{k}^{*}=\left(V_{k}^{*}, E_{k}^{*}\right)$, each assigned a weight $w_{k^{\prime}}^{\prime} k=1, \cdots, m$. Then, if $G^{*}$ is a maximal $r$-regular subnetwork and if for any node $i \in V \backslash V^{*}$, there are at least $r+1$ nodes $j \in V^{*}$ such that $(i, j) \in E$ and $w_{i} \geq w_{j}, x^{*}$ is a Nash equilibrium for the game, with $x_{i}^{*}=\tilde{w}^{2} / w_{k}^{\prime 2}$ for all $i \in V_{k}^{*}, k=1, \cdots, m$, and $x_{i}^{*}=0$ for all $i \in V \backslash V^{*}$, where $1 / \tilde{w}^{2}=\Sigma_{k} m_{k} / w_{k}^{\prime 2}$, with $m_{k}$ being the size of $V_{k}^{*}$.

Proof. Since $G^{*}$ supports $x^{*}, x_{i}^{*}>0$ for all $i \in V^{*}$ and $x_{i}^{*}=0$ for all $i \in V \backslash V^{*}$. Then $p_{i}\left(x^{*}\right)=$ $\Sigma_{j} \tilde{A}_{i, j} x_{j}^{*}=\Sigma_{j \in V_{k}^{*}} \tilde{A}_{i, j} x_{j}^{*}=w_{k}^{\prime 2} \Sigma_{(i, j) \in E_{k}^{*}} x_{j}^{*}$ for all $i \in V_{k}^{*}$. Let $w_{k}^{\prime 2} \Sigma_{(i, j) \in E_{k}^{*}} x_{j}^{*}=\lambda^{*}$ for all $i \in V_{k}^{*}$. By adding the equations for all $i \in V_{k}^{*}$, we obtain $w_{k}^{\prime 2}(r+1) \Sigma_{j \in V_{k}^{*}} x_{j}^{*}=m_{k} \lambda^{*}$. By adding the 
latter equations for all $k$, we then obtain $\lambda^{*}=(r+1) \tilde{w}^{2}$. It follows that $w_{k}^{\prime 2} \Sigma_{(i, j) \in E_{k}^{*}} x_{j}^{*}=\lambda^{*}$ for all $i \in V_{k}^{*}$ with $x_{j}^{*}=\tilde{w}^{2} / w_{k}^{\prime 2}$ for all $j \in V_{k}^{*}$. Note that for any node $i \in V \backslash V^{*}$,

$$
\begin{aligned}
p_{i}\left(x^{*}\right) & =\Sigma_{j} \tilde{A}_{i, j} x_{j}^{*}=\Sigma_{k} \Sigma_{j \in V_{k}^{*}} \tilde{A}_{i, j} x_{j}^{*} \\
& =\Sigma_{k} \Sigma_{j \in V_{k}^{*},(i, j) \in E} w_{i} w_{j} x_{j}^{*} .
\end{aligned}
$$

Since there are at least $r+1$ nodes $l \in V^{*}$ such that $(i, l) \in E$ and $w_{i} \geq w_{l}$,

$$
\begin{aligned}
p_{i}\left(x^{*}\right) & \geq \Sigma_{k} \Sigma_{l \in V_{k}^{*},(i, l) \in E} w_{i} w_{l} x_{l}^{*} \\
& \geq \Sigma_{k} \Sigma_{l \in V_{k}^{*},(i, l) \in E} w_{l}^{2} x_{l}^{*} \\
& =\Sigma_{k} \Sigma_{l \in V_{k}^{*},(i, l) \in E} \tilde{w}^{2} \\
& \geq(r+1) \tilde{w}^{2}=\lambda^{*} .
\end{aligned}
$$

By Theorem 4.1, $x^{*}$ is a Nash equilibrium.

Note that in Theorems 4.2, 4.3,4.4, 4.5, the conditions that for any node $i \in V \backslash V^{*}$, there must be at least $r+1$ nodes $j \in V^{*}$ such that $(i, j) \in E$ and $w_{i} \geq w_{j}$ are sufficient but not necessary. In general, the inequality conditions in Theorem 4.1 may need to be tested. In any case, as an example, consider the network in Fig. 5. Assume that the nodes in $\{1,2,3,4\}$ are assigned a weight $w_{1}^{\prime}=3,\{5,9,10\}$ assigned $w_{2}^{\prime}=2,\{7,13,14\}$ assigned $w_{3}^{\prime}=2,\{6,11,12\}$ assigned $w_{4}^{\prime}=1$, and $\{8,15,16\}$ assigned $w_{5}^{\prime}=1$. Assume that additive weights are used for the calculation of the social contacts. Consider the maximal 2-regular subnetwork formed by $\{5,6,7,8,9,10,11,12,13,14,15,16\}$. Then, $\bar{w}=1 /\left(3 / w_{2}^{\prime}+3 / w_{3}^{\prime}+3 / w_{4}^{\prime}+3 / w_{5}^{\prime}\right)=1 / 9$. Let $x_{1}^{*}=x_{2}^{*}=x_{3}^{*}=x_{4}^{*}=0, x_{5}^{*}=x_{9}^{*}=x_{10}^{*}=\bar{w} / w_{2}^{\prime}=1 / 18, x_{7}^{*}=x_{13}^{*}=x_{14}^{*}=\bar{w} / w_{3}^{\prime}=1 / 18, x_{6}^{*}=x_{11}^{*}=$ $x_{12}^{*}=\bar{w} / w_{4}^{\prime}=2 / 18$, and $x_{8}^{*}=x_{15}^{*}=x_{16}^{*}=\bar{w} / w_{5}^{\prime}=2 / 18$. Then, for any node $i \in\{1,2,3,4\}$, there are 3 nodes $j \in\{5,6,7,8,9,10,11,12,13,14,15,16\}$ such that $i$ and $j$ are connected and $w_{i} \geq w_{j}$ since $w_{i}=3$ while $w_{j}=1$ or 2 . Therefore, by Theorem $4.4, x^{*}$ is a Nash equilibrium, with the total amount of social contacts minimized to $\lambda^{*}=(r+1) \bar{w}=1 / 3$.

In previous sections, we have discussed the rigidity, flexibility, and fragility of distancing strategies for social distancing games. These properties all depend on the structures of the supporting subnetworks. Therefore, Theorems 3.1, 3.2, 3.3, and 3.4 can all be extended straightforwardly to the equilibrium strategies for weighted social distancing games since the weighting schemes we have considered do not change the network structures. In particular, for a supporting subnetwork, if the same weight is assigned to the nodes of each component of the subnetwork, as we have assumed in the above discussion, we can show that (a) an equilibrium strategy of the weighted game, with either additive or multiplicative weights, must be strongly rigid if its supporting subnetwork is a maximal independent set; (b) it is flexible if its supporting subnetwork is a maximal $r$-regular subnetwork, $r>0$; (c) it is weakly rigid if its supporting network is a maximal $r$-regular subnetwork whose components are all minimal $r$-regular subnetworks, $r>0$; and (d) it is fragile if its supporting subnetwork is an $r$-regular subnetwork, $r>0$, which 
is connected but not minimal, or disconnected but at least one of its components is not minimal. The proofs follow almost the same steps as in the proofs for Theorems 3.1, 3.2, 3.3, and 3.4. We will not elaborate further.

\section{Concluding remarks}

Much work has been done in the past on social distancing as a behavioral factor affecting the development of an infectious disease, but research on how to effectively practice and manage social distancing is lacking. The latter can in fact be an even more important research subject, for the effectiveness of social distancing would be greatly undermined if without effective practicing. We have considered a social distancing problem for how a population, when in a world with a network of social sites, chooses some sites to visit or stay while avoiding or closing down some others so that the social contacts of the population across the network can be minimized. We have modeled this problem as a population game with the Nash equilibrium of the game corresponding to an optimal strategy for choosing among given social sites for social distancing. The social sites that are selected at equilibrium form a subnetwork, which we have recognized as a structural support for the equilibrium strategy.

We have shown that a large class of equilibrium strategies can be supported by a maximal $r$-regular subnetwork. The latter includes many well studied network types, which are easy to identify or construct, and can be completely disconnected for the most strict isolation (with $r=0$ ) or allow certain degrees of connectivity for more flexible distancing (with $r>0$ ). We have derived the equilibrium strategies on (a) maximal 0-regular subnetworks known as maximal independent sets; and (b) maximal $r$-regular subnetworks for $r>0$. We have also extended these results to weighted networks and derived equilibrium strategies with additive and multiplicative weights on (c) maximal weighted 0-regular subnetworks; and (d) maximal weighted $r$-regular subnetworks for $r>0$.

We have introduced the concepts of rigidity, flexibility, and fragility of a social distancing strategy, and analyzed these properties for strategies on different types of $r$-regular subnetworks. We have shown that (a) strategies on maximal 0-regular subnetworks are strongly rigid; (b) strategies on maximal $r$-regular subnetworks are flexible for all $r>0$; (c) strategies on maximal $r$-regular subnetworks whose components are minimal $r$-regular subnetworks are weakly rigid for all $r>0$; and (d) strategies on maximal $r$-regular subnetworks with at least one non-minimal $r$-regular component are fragile for all $r>0$. These results apply to both weighted and unweighted networks.

Our work is a step towards developing a general theoretical and computational framework for the study of social distancing in networked social environments. It is however open for many further research efforts. First of all, new dynamic epidemic models can be built for populations distributed over social networks as determined by the equilibrium strategies for the social distancing games. Different from those for arbitrarily mixed populations, such models may provide more realistic estimates on various epidemic proper- 
ties such as contact rates, transmission rates, and hence the infectious rates, etc. Dynamic models on structured populations are always preferred because populations are always heterogeneous in terms of physical or social proximities [50].

The types of supporting subnetworks we have discussed are $r$-regular networks because they are easy to identify or construct, yet cover a broad range of networks with varying degrees of connectivities. However, in reality, the supporting subnetworks may not always be so "regular". The population may prefer other types of network structures such as spanning trees, extended stars, or small-world networks, all with low degrees of connectivities [51]. It would be interesting to find the equilibrium conditions for distancing strategies on such networks, including related distancing properties such as rigidity, flexibility, and fragility of the strategies.

When defining social distancing games, we have assumed that the population is homogeneous, and all the individuals can therefore take the same optimal strategy at equilibrium. However, in reality, the population is more likely to be heterogeneous, with possible subpopulations divided either geographically or demographically and hence different in distancing preferences. For example, populations in different geographic locations may prefer going to social sites close to their own regions; different age groups may have different social activities and hence different social sites to visit. The current model may be extended to such populations by defining a more general multi-player population game with the individuals in different subpopulations as different players against the population.

Social distancing brings economic costs or social sacrifices. Different distancing strategies may have different social or economic consequences $[2,6,7,13]$. Reducing social contacts is a main goal as far as fighting a pandemic is concerned, but keeping certain levels of social or economic activities can be important as well especially during a possibly long period of recovery from the pandemic [3-5]. Therefore, there must be a tradeoff between choosing rigid and flexible distancing strategies. A cost-benefit function may be defined as a function of the amount of social contacts of any distancing strategy. The social distancing game can then be generalized to find an optimal distancing strategy that maximizes distancing benefits at lowest possible social or economic costs.

In this paper, we have focused on distancing strategies on maximal $r$-regular subnetworks, which are supposed to be easy to identify or construct $[52,53]$. Still, when a large network is given, it takes time to find such a subnetwork. It is well known that the problem to find a maximum 0-regular induced subgraph of a given graph, i.e., a maximum independent set, is NP-hard [54]. The problem to find a maximum 1-regular induced subgraph of a given graph, known as the maximum strong matching problem, is also NP-hard $[55,56]$. In fact, it has been proved that the problem to find a maximum $r$-regular induced subgraph of a given graph is NP-hard for any $r \geq 0[57,58]$. Fortunately, for our purpose, we do not have to find a maximum $r$-regular subnetwork. A maximal $r$-regular subnetwork would be sufficient to support an equilibrium distancing strategy, which is relatively easier to find than a maximum one.

Finally, we would also like to point out that the social networking game as described 
in the first section of the paper has been studied previously as a population game [1]. It becomes relevant when social distancing comes into play, for the latter can be considered exactly as a complementary game to the former. The social networking game has been formulated in different forms and for different purposes in the past. It has been used for the solution of the maximum clique problem, since the maximum cliques of a given network correspond to the maximum payoff equilibria of the social networking game on this network [59]. It has also been used for modeling the evolution of allele selection in genetic research as an evolutionary game on genetic selection graphs [60]. The work on the social distancing game on a network in this paper benefits from many ideas and results coming out from these previous studies.

\section{Acknowledgments}

The author would like to thank Claus Kadelka, Audrey McCombs, and Rana Parshad to share their work with the author, and thank the anonymous reviewers for carefully reading the paper and providing valuable comments and suggestions. The author would also like to acknowledge the support on this work from the Simons Foundation through the Mathematics and Physical Sciences Collaboration Grants for Mathematicians.

\section{References}

[1] Wang, M., Zhou, W., and Wu, Z., Equilibrium distributions of populations of biological species on networks of social sites, Journal of Biological Dynamics, 13: 74-98, 2019.

[2] Chakradhar, S., To fight coronavirus spread, the U.S. may extend 'social distancing' measures, but it comes at a cost, STAT News, February 17, 2020.

[3] Kissler, S., Tedijanto, C., Goldstein, E., et al., Projecting the transmission dynamics of SARS-CoV-2 through the post-pandemic period, Science, online April 14, 2020, doi:10.1126/science.abb5793.

[4] Long, N., From social distancing to social containment: reimagining sociality for the coronavirus pandemic, Medicine Anthropology Theory, ISSN 2405-691x (submitted), 2020.

[5] Mann, C., Pandemics leave us forever altered - What history can tell us about the long-term effects of the coronavirus, The Atlantic: IDEAS, June 2020.

[6] Miller, G., Social distancing prevents infections, but it can have unintended consequences, Science News, March 16, 2020.

[7] Thunstrome L., Newbold, S., Finnoff, D., et al., The benefits and costs of using social distancing to flatten the curve for COVID-19, Journal of Benefit Cost Analysis, online March 27, 2020, doi:10.2139/ssrn.3561934.

[8] Krause, J. and Ruxton, G., Living in Groups, Oxford University Press, 2002.

[9] Alcock, J., Animal Behavior: An Evolutionary Approach, Sinauer Associates Inc., 2009.

[10] Combs, S., These wild animals also practice social distancing to avoid getting sick, National Geographic News, March 24, 2020.

[11] Hatchett, R., Mecher, C., and Lipsitch, M., Public health interventions and epidemic intensity during the 1918 influenza pandemic, PNAS, 104: 7582-7587, 2007. 
[12] Roth, D., Henry, B., Social distancing as a pandemic influenza prevention measure, National Collaborating Center for Infectious Diseases, Winnipeg, MB, Canada, July, 2011.

[13] Fenichel, E., Economic considerations of social distancing and behavioral based policies during an epidemic, Journal of Health Economics, 32: 440-451, 2013.

[14] Ahmed, F., Zviedrite, N., and Uzicanin, A., Effectiveness of workplace social distancing measures in reducing influenza transmission: A systematic review, BMC Public Heath, 8: 518-530, 2018.

[15] Faherty, L., Schwartz, H., Ahmed, F., Zheteyeva, Y., Uzicanin, A., and Uscher-Pines, L., School and preparedness officials' perspectives on social distancing practices to reduce influenza transmission during a pandemic: Considerations to guide future work, Preventive Medicine Reports, 14: 100871, 2019.

[16] Wilder-Smith, A. and Freedman, D, Isolation, quarantine, social distancing and community containment: Pivotal role for old-style public heath measures in the novel coronavirus (2019nCoV) outbreak, Journal of Travel Medicine, 2020: 1-4.

[17] Eubank, S., Guclu, H., Anil Kumar, V., Marathe, M., Srinivasan, A., Toroczkai, Z., and Wang, N., Modeling disease outbreaks in realistic urban social networks, Nature, 429: 180-184, 2004.

[18] Del Valle, S., Hethcote, H., Hyman, J., and Castillo-Chavez, C., Effects of behavioral changes in a smallpox attack model, Mathematical Biosciences, 195: 238-251, 2005.

[19] Glass, R., Glass, L., Beyeler, W., and Min, H., Targeted social distancing design for pandemic influenza, Emerging Infectious Diseases, 12: 1671-1681, 2006.

[20] Meyers, L., Contact network epidemiology: Bond percolation applied to infectious disease prediction and control, Bulletin of American Mathematics Society, 44: 63-86, 2007.

[21] Caley, P., Philp, D., and McCracken, K., Quantifying social distancing arising from pandemic influenza, Journal of Royal Society, Interface, 5: 631-639, 2008.

[22] Kelso, J., Milne, G., and Kelly, H., Simulation suggests that rapid activation of social distancing can arrest epidemic development due to a novel strain of influenza, BMC Public Health, 9: 1, 2009.

[23] Funk, S., Salathe, M., and Jansen, V., Modeling the influence of human behavior on the spread of infectious diseases: A review, Journal of Royal Society, Interface, 7: 1247-1256, 2010.

[24] Reluga, T., Game theory of social distancing in response to an epidemic, PLoS Computational Biology, 6: c1000793, 2010.

[25] Chen, F., Jiang, M., Rabidoux, S., and Robinson, S., Public avoidance and epidemics: Insights from an economic model, Journal of Theoretical Biology, 278: 107-119, 2011.

[26] Valdez, L., Buono, C., Macri, P., and Braunstein, L., Intermittent social distancing strategies for epidemic control, Physics Review E, 85: 036108, 2012.

[27] Bhattacharyya, S. and Reluga, T., Game dynamic model of social distancing while cost of infection varies with epidemic burden, IMA Journal of Applied Mathematics, 84: 23-43, 2019.

[28] Eubank, S., Eckstrand, I., Lewis, B., Venkatramanan, S., Marathe, M., and Barrett, C., Commentary on Ferguson, et al., "Impact on non-pharmaceutical interventions (NPIs) to reduce COVID-19 mortality and healthcare demand", Bulletin of Mathematics Biology, DOI: https://doi.org/10.1007/s11538-020-00726-x, 2020.

[29] Ferguson, N., Laydon, D., Nadjati-Gilani, N., et al., Impact of non-pharmaceutical interventions (NPIs) to reduce COVID-19 mortality and healthcare demand, Imperial College COVID19 Response Team, London, March 16, 2020.

[30] Hellewell, J., Abbott, S., Gimma, A., et al., Feasibility of controlling COVID-19 outbreaks by isolation of cases and contacts, The LANCET Global Health, 8: E488-E496, 2020.

[31] Kiesha, P., Liu, Y., Russell T., et al., The effect of controlling strategies to reduce social mixing 
on outcomes of the COVID-19 epidemic in Wuhan, China: A modeling study, LANCET PUBLIC Health, DOI: https: / doi.org/10.1016/S2468-2667(20)30073-6, 2020.

[32] McCombs, A. and Kadelka, C., A model-based evaluation of the efficacy of COVID-19 social distancing, testing and hospital triage policies, medRxiv: doi: https://doi.org/10.1101/2020.04.20.20073213, 2020.

[33] Sanche, S., Lin, Y., Xu, C., et al., The Novel coronavirus, 2019-nCoV, is highly contagious and more infectious than initially estimated, arXir.org: 2002, 03268, 2020.

[34] Bondy, A. and Murty, U., Graph Theory, Springer, 2008.

[35] Newman, M., Networks, Oxford University Press, 2018.

[36] Reluga, T. and Galvani, A., A general approach for population games with applications to vaccination, Mathematical Biosciences, 230: 67-78, 2011.

[37] Reluga, T., Equilibria of an epidemic game with piecewise linear social distancing cost, Bulletin of Mathematical Biology, 75: 1961-1984, 2013.

[38] Barrett, C., Eubank, S., Anil Kumar, V., and Marathe, M., Understanding large-scale social and infrastructure Networks: A simulation-based approach, SIAM News, 37, May, 2004.

[39] Eubank, S., Network based models of infectious disease spread, Japanese Journal of Infectious Diseases, 58: S9-S13, 2005.

[40] Eubank, S., Anil Kumar, V., Marathe, M., Srinivasan, A., and Wang, N., Structure of social networks and their impact on epidemics, DIMACS Series in Discrete Mathematics and Theoretical Computer Science, 70: 181-213, 2006.

[41] Barrett, C., Bisset, K., Eubank, S., Feng, X., Marathe, M., EpiSimdemics: An efficient algorithm for simulating the spread of infectious disease over large realistic social networks, SC08: Proceedings of the 2008ACM/IEEE Conference on Supercomputing, 1-12, 2008.

[42] Meyers, L., Newman, M., and Pourbohloul, B., Predicting epidemics on direct contact networks, Journal of Theoretical Biology, 240: 400-418, 2006.

[43] Volz, E. and Meyers, L., Susceptible-infected-recovered epidemics in dynamic contact networks, Proceedings of the Royal Society B, 274: 2925-2933, 2007.

[44] Volz, E. and Meyers, L., Epidemic thresholds in dynamic contact networks, Journal of the Royal Society, Interface, 6: 233-241, 2009.

[45] Bansal, S., Read, J., Pourbohloul, B., Meyers, L., The dynamic nature of contact networks in infectious disease epidemiology, Journal of Biological Dynamics, 4: 478-489, 2010.

[46] Weibull, J. W., Evolutionary Game Theory, The MIT Press, 1995.

[47] Hofbauer, J. and Sigmund, K., Evolutionary Games and Population Dynamics, Cambridge University Press, 1998.

[48] Fletcher, R., Practical Optimization, Wiley, 2000.

[49] Nocedal, J. and Wright, S., Numerical Optimization, Springer, 2006.

[50] Brauer, F. and Castillo-Chavez, C., Mathematical Models in Population Biology and Epidemiology, 2nd Edition, Springer 2011.

[51] Lewis, T., Network Science: Theory and Applications, Wiley, 2009.

[52] Cormen, T., Leiserson, C., Rivest, R., and Stein, C., Introduction to Algorithms, Third Edition, The MIT Press, 2009.

[53] Erickson, J., Algorithms, Independent Publisher, 2019.

[54] Garey, M. and Johnson, D., Computers and Intractability: A Guide to the Theory of NPCompleteness, Freeman, 1979.

[55] Stockmeyer, L. and Vazirani, V., NP-completeness of some generalizations of the maximum matching problem, Information Processing Letters, 15: 14-19, 1982.

[56] Cameron, K., Induced matchings, Discrete Applied Mathematics, 24: 97-102, 1989. 
[57] Cardoso, D., Kaminski, M., and Lozin, V., Maximum k-regular induced subgraphs, Rutcor Research Report (RRR), 3, 2006.

[58] Gupta, S., Raman, V., and Saurabh, S, Fast exponential algorithms for maximum $r$-regular induced subgraph problems, in S. Arun-Kumar and N. Garg (Eds): FSTTCS 2006: Lecture Notes in Computer Science, 4337: 139-151, 2006.

[59] Bomze, I., Evolution towards the maximum clique, Journal of Global Optimization, 10: 143-164, 1997.

[60] Vickers, G. and Cannings, C., On the number of stable equilibria in a one-locus, multi-allelic system, Journal of Theoretical Biology, 131: 273-277, 1988. 